\title{
Indirect geometry measurement for laser chemical machin- ing using a model-based signal processing approach
}

\author{
Merlin Mikulewitsch ${ }^{1}$, Dirk Stöbener ${ }^{1,2}$, Andreas Fischer ${ }^{1}$ \\ 1 University of Bremen, Bremen Institute for Metrology, Automation and Quality Science (BIMAQ), Lin- \\ zer Str. 13, 28359 Bremen, Germany \\ 2 University of Bremen, MAPEX Center for Materials and Processes, Germany \\ m.mikulewitsch@bimaq.de
}

\begin{abstract}
Summary:
The optical geometry measurement of submerged micro-surfaces in chaotic fluid environments, e.g., for electric discharge machining (EDM) or laser chemical machining (LCM), is challenging when the specimen features high aspect ratios and steep surface gradients. To avoid reflection-caused artifacts at steep gradients, fluorescent molecules are added to the fluid and the geometry of the fluid layer is measured using a confocal fluorescence microscope. A model-based signal processing enables a robust, indirect measurement in fluid layers $>1 \mathrm{~mm}$, since it is capable to cope with process-inherent bubbles and surface gradients up to $85^{\circ}$.
\end{abstract}

Keywords: micro geometry, optical measurement, in situ, fluorescence, signal modeling

\begin{abstract}
Introduction
Laser chemical machining (LCM) uses an electrolytic fluid and localized heating by a laser to generate a material removal in submerged workpieces. It produces microstructures with high aspect ratios, high surface gradients and small edge radii. In contrast to competing processes such as micro-milling or laser ablation there is no thermal stress induction or tool wear [1]. However, the in situ conditions hinder conventional optical geometry measurement methods.
\end{abstract}

Interferometric methods such as white light interferometry suffer from measurement deviations caused by thermal gradients and refractive index fluctuations in the fluid [2]. Confocal microscopy is prone to artifacts caused by the high surface angles and curvatures [3] typically produced with the LCM process. However, an indirect measurement approach using confocal fluorescence microscopy is promising for in situ application, since it does not capture the light reflected by the specimen, but the light emitted by a fluorescent liquid covering it. The detected fluorescence signal $S(z)$ can be limited to a small volume around the focal plane of the objective by axial light sectioning produced by confocal microscopy, where a pinhole, confocal to the objective lens, attenuates light far outside the focal plane. This way, a signal is only detected when the so-called confocal volume intersects the fluorescent fluid. The surface geometry is then determined by the change of the fluorescence signal produced by pointwise scanning of the confocal volume from the fluid surface to the specimen surface.

The principle was successfully used on metallic microspheres with high curvatures by coating their surface with a thin fluorescent film $<100 \mathrm{~nm}$ [3]. It was shown that a measurement was possible even at angles $>75^{\circ}$ from the surface normal with a lateral resolution comparable to conventional confocal microscopy without causing artifacts. The influence of gas bubbles and high surface inclinations on the measurement in thick fluid layers $>1 \mathrm{~mm}$ present during LCM has not yet been studied and is the aim of this work.

\section{Model-based indirect measurement method for thick fluid layers}

To determine the specimen surface position $z_{0}$ with micrometer precision in thick fluid layers, a model-based evaluation of the fluorescence signal $S(z)$ is required [4]. The signal model is based on a simplified confocal volume function (CVF) in the shape of a 3D-Gaussian function. It represents the spatial distribution of the contributions of all infinitesimal volume elements to the total fluorescence signal. The signal function $S(z)$ at the position $z$ is obtained by weighting the CVF with a depth dependent absorption term and integrating it over all spatial dimensions [4]. To cope with small deviations due to inclined surfaces, the model was heuristically extended by two additional terms, resulting in 


$$
\begin{aligned}
S(z)= & S_{0} e^{\epsilon\left(z-z_{1}\right)}\left[\operatorname{erf}\left(\frac{z-z_{0}}{2 \Xi}+\epsilon \Xi\right)-\operatorname{erf}\left(\frac{z-z_{1}}{2 \Xi}+\epsilon \Xi\right)+K_{1} .\right. \\
& \left.\left(\operatorname{erf}\left(\frac{z-z_{2}}{2 \xi}+\epsilon \xi\right)-\operatorname{erf}\left(\frac{z-z_{1}}{2 \xi}+\epsilon \xi\right)\right)\right]+K_{2} e^{-\frac{\left(z-z_{2}\right)^{2}}{2 \sigma^{2}}},
\end{aligned}
$$

with $\epsilon$ being the attenuation coefficient, $\xi / \Xi$ confocal volume parameters and $S_{0}, K_{1,2}, \sigma$ and $z_{2}$ weighting parameters. The desired position $z_{0}$ (specimen surface) is finally obtained by using the model function $S(z)$ for a non-linear leastsquares approximation of the measured data.

\section{Results}

The fluorescence intensity signals along a line on the inclined surface of a submerged specimen were measured for the three inclinations $35^{\circ}, 65^{\circ}$ and $85^{\circ}$. The specimen surface positions $z_{0}$ resulting from the model-fit using Eq. (1) are shown in Fig. 1 (left). To determine the influence of the surface angle on the indirect measurement, the deviations $\delta$ from a linear fit of the surface positions were calculated. Although the standard deviation of $\delta$ increases with the angle, a comparison with a reference measurement using conventional confocal microscopy shows an equal magnitude as the indirect measurement. In contrast to the reference measurements however, no artifacts in form of systematic measurement deviations were observed during indirect measurement for surface angles up to $85^{\circ}$.
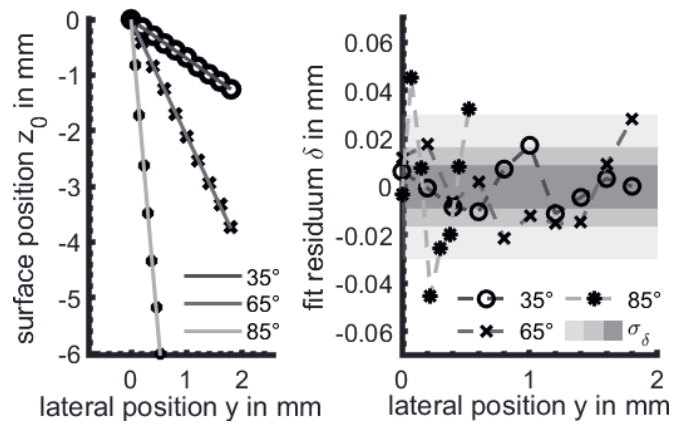

Fig. 1. Left: Surface positions for three different inclinations (with linear fit). Right: Fit residuum $\delta$ with standard deviations (shaded areas).

Since gas bubbles occur in the LCM process and may cross the optical path of the measurement system, the influence on the indirect measurement needs to be determined. For that purpose, a bubbly fluid environment was emulated by chemical reactions of phosphoric acid and a nonpassivating metallic specimen material. The fluorescence intensity signals for two different bubble densities in the optical path are shown in Fig. 2. The fluorescence signal exhibits an increased noise with higher bubble density, particularly near the fluid surface. The determination of the surface position $z_{0}$ using the model-based signal approach is possible nonetheless, even with the increased signal noise. The result is merely an increased uncertainty of $z_{0}$, see measurements on a flat surface shown in Fig. 3.
In conclusion, the indirect geometry measurement method is suitable for in situ measurements in thick and contaminated fluid layers as they appear in the LCM or EDM process environments. In particular, it enables measurements on surface angles up to $85^{\circ}$.

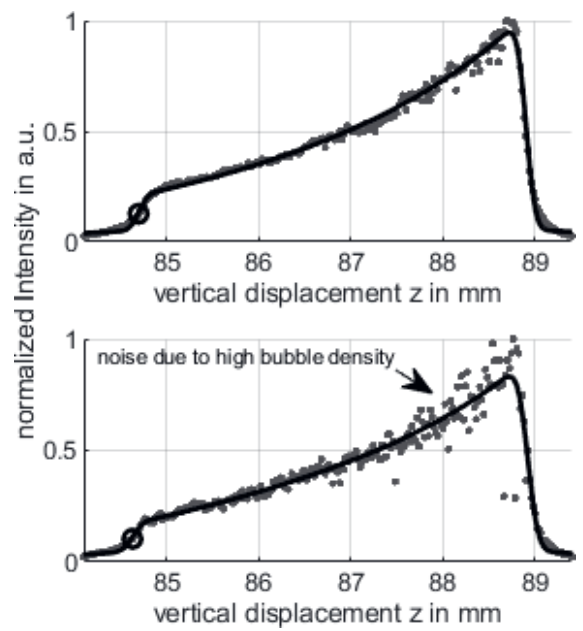

Fig. 2. Fluorescence intensity signal and model fit for a low (top) and a high (bottom) gas bubble density in the optical path (circle: $z_{0}$ ).

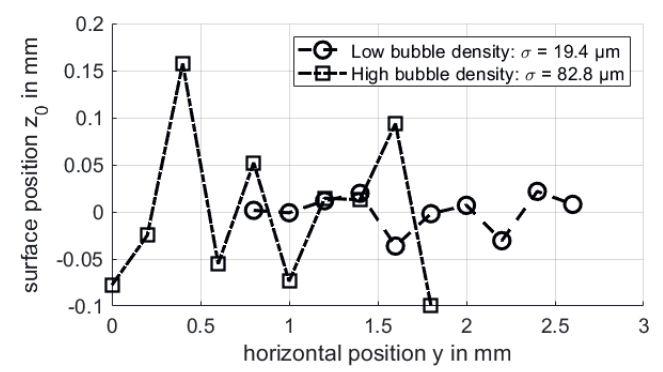

Fig. 3. Surface positions $z_{0}$ along the $y$-axis on a level surface. The standard deviation $\sigma$ of the surface position $z_{0}(y)$ increases with bubble density.

Funding DFG - SFB747.

\section{References}

[1] H. Messaoudi, M. Mikulewitsch, D. Brand, A. von Freyberg, A. Fischer, Removal behavior and output quality for laser chemical machining of tool steels, Manuf. Rev. 6, 13 (2019); doi: 10.1051/mfreview/2019015

[2] C. Gerhard, F. Vollertsen, Limits for interferometric measurements on rough surfaces in streaming inhomogeneous media, Prod. Eng. Res. Devel. 4, 141-146 (2010); doi: 10.1007/s11740-010-0224-7

[3] J. Liu, C. Liu, J Tan, B. Yang, T. Wilson, Super-aperture metrology: overcoming a fundamental limit in imaging smooth highly curved surfaces, J. Microsc. 261, 300-306 (2016); doi: 10.1111/jmi.12334

[4] M. Mikulewitsch, A. von Freyberg, and A. Fischer, Confocal fluorescence microscopy for geometry parameter measurements of submerged micro-structures. Opt. Lett. 44, 1237-1240 (2019); doi: 10.1364/OL.44.001237 